which is much lower than the surface fields required for pulsars and allows the extremely rapid rotation to persist for millions of years.

Typical time scales which Davidson et al. expect to be associated with the motions of the components of their model range from the pulsar period, roughly $0.003 \mathrm{~s}$, to the time scale for dynamic equilibrium, roughly $3,000 \mathrm{~s}$. The orbital period of the gas is $30 \mathrm{~s}$, and its cooling time is roughly $0.3 \mathrm{~s}$, so that it must indeed be continuously excited for the system to be stable.

Most of the objections to this model are certainly no more severe than those which apply to other models for Sco X-1. Indeed, with the exception of the rather low magnetic field, the estimated parameters agree well with observations of this and other sources, and the cocoon seems a suitable source of erratic variations of the kind detected. Two crucial tests seem relevant. One or two such objects could be detected without a drastic re-thinking of the theory, but many more sources in the Sco X-1 mould would rapidly become a surfeit. And if Sco X-1 itself does show a continuation of the decline in activity and, possibly, temperature which has been hinted at by some recent observations, then it would seem to be too short lived for the Davidson et al. model-this would certainly be a new development, because the downfall of most models of the object so far has been their implausibly short time scales compared with the observed lifetime of Sco X-1.

\section{MINICELLS \\ Exploifing Cytochalasin}

from our Cell Biology Correspondent

Although minicells, small enucleate Escherichia coli, have been used occasionally by molecular biologists, it seems safe to say that they became available too late in the game. Many questions, which might with comparative ease have been answered by experiments with minicells, had already been solved by hard work and ingenuity with nucleate $E$. coli. But animal virologists look to be more fortunate for it seems, if the experiments of Prescott, Kates and Kirkpatrick (J. Mol. Biol., 59, 505; 1971) are anything to go by, that enucleate cells obtained by exposing nucleate cells in culture to cytochalasin B can be exploited to answer many outstanding questions.

Prescott and his colleagues have used enucleate $L$ cells produced in this way to prove quite unambiguously that the uncoating and replication of vaccinia virus does not depend on the presence of a host cell nucleus. After exposing $\mathrm{L}$ cells to cytochalasin $\mathrm{B}$, and in some experiments to actinomycin $\mathrm{D}$ as well to inhibit any RNA synthesis that the cyto- chalasin B might induce, and then washing the cultures and incubating them for $2 \mathrm{~h}$ in medium they added vaccinia virus and followed the fate of the infected nucleate and enucleate cells by light microscopy and autoradiography. Typical "factories" synthesizing vaccinia DNA develop in the enucleate cells and so the suggestion, originally made by Joklik in 1964, that the uncoating of vaccinia virus depends on a function coded by a host cell gene and activated by infection, has been neatly eliminated.

Joklik's suggestion stemmed, of course, from the finding that after the removal of the lipoprotein outer coat of vaccinia virus, the second step in its uncoating, the disruption of the inner "core" protein coat is specifically blocked by inhibitors of RNA and protein synthesis. This indicated that the synthesis and translation of messenger RNA was essential for the second stage of uncoating and in 1964 it seemed quite plausible that the gene(s) in question might well be cellular rather than viral. By 1967, however, Kates and McAuslan had shown that vaccinia cores with their protein coat intact can make and release messenger and Joklik's idea was beginning to look unnecessarily complex. Prescott's results are not altogether unexpected therefore and the chief interest in what he and his colleagues have to say lies in the method they have used.

Clearly, hypotheses which implicate directly the host genome in the replication of other animal viruses might be put to the test with enucleate cells. It would, for example, be interesting to know how far the replication of RNA tumour viruses proceeds in such cells. For that might reveal whether or not the stable association between a DNA provirus, which, as Hatanaka et al. have just shown (Proc. US Nat. Acad. Sci., $68,1844 ; 1971)$, is probably made in the cytoplasm, and the host genome is essential for replication of these viruses.

\title{
Transfer RNA Precursor Sequenced
}

IN next Wednesday's Nature New Biology, Altman and Smith describe their latest investigations of the tyrosine transfer RNA precursor molecule of Escherichia coli and its maturation into tyrosine transfer RNA; their work is a remarkable example of the pitch of sophistication which has been reached in analyses of bacteria.

As Altman recently reported in Nature New Biology (229, 19 ; 1971) transfer RNAs, like ribosomal and probably messenger RNAs, are transcribed as part of a larger precursor molecule which is matured by cleavage. What Altman and Smith have now done is analyse the base sequence of the precursor of a tyrosine transfer RNA, prove that mutations which alter the amount of the mature RNA produced are located in a region of the precursor close to the cleavage point and detect a nucleolytic activity in extracts of $E$. coli which in vitro matures the precursor.

Obtaining strains which accumulate precursor involved a series of elegant genetic manipulations; with these achieved Altman and Smith obtained enough precursor to analyse its base sequence and they found several striking features. First, bases which, in mature transfer RNA, are modified are, in the precursor, unmodified. Second, it seems that the CCA sequence at the $3^{\prime}$ terminal end of the mature molecule is present in the precursor, contrary perhaps to expectation. Third, many of the oligonucleotides obtained after partial digestion of the precursor with $T_{1}$ ribonuclease correspond to those obtained after partial digestion of the mature molecule. This situation suggests that even in the precursor the secondary structure of the mature tRNA sequence has a clover leaf secondary structure. Finally, the $5^{\prime}$ terminal residue of the precursor is pppGp, which suggests that transcription in vivo as in vitro is initiated with a $5^{\prime}$ triphosphate purine nucleotide.

Altman and Smith then sequenced the precursor specified by a mutated tyrosine transfer RNA gene, which in vivo matures unusually rapidly. To do this, of course, they had to introduce other mutations which caused this precursor to accumulate. The sequence revealed that the mutation which leads to a rapidly maturing precursor changes a base four nucleotides removed from the cleavage point. Moreover, other mutations which cause the production of reduced amounts of mature transfer RNA but no accumulation of precursor also change bases close to the cleavage point. For these reasons Altman and Smith suggest that both the base sequence and the secondary structure at the cleavage point probably affect the rate and the precise point of cleavage and they propose models of the structure of the precursor which might account for these factors.

Finally, they have succeeded in detecting in extracts of $E$. coli a nucleolytic activity which in vitro cleaves the precursor at the correct point and yields a transfer RNA fragment with the same $5^{\prime}$ terminus as transfer RNA matured in vivo. This finding proves that modification of certain bases in the transfer RNA segment of the precursor is not essential for proper maturation. Indeed, modification may well occur in vivo after the precursor has been cleaved. 